\title{
Identification of Key Genes and miRNA-mRNA Regulatory Pathways in Bronchopulmonary Dysplasia in Preterm Infants by Bioinformatics Methods
}

\author{
Tong Sun ${ }^{1}$, Haiyang $\mathrm{Yu}^{1}$, and Jianhua $\mathrm{Fu}^{1}$ \\ ${ }^{1}$ Shengjing Hospital of China Medical University
}

September 11, 2020

\begin{abstract}
Background: BPD remains a severe respiratory complication of preterm infants in NICUs. However, its pathogenesis has been unclear. Bioinformatics analysis, which can help us explore genetic alternations and recognize latent diagnostic biomarkers, has recently promoted the comprehension of the molecular mechanisms underlying disease occurrence and development. Methods: In this study, we identified key genes and miRNA-mRNA regulatory networks in BPD in preterm infants to elucidate the pathogenesis of BPD. We downloaded and analyzed miRNA and gene expression microarray datasets from the Gene Expression Omnibus database (GEO). Differentially expressed miRNA (DEMs) and differentially expressed genes (DEGs) were obtained through NetworkAnalyst. We performed pathway enrichment analysis using the Database for Annotation, Visualization and Integrated Discovery program (DAVID), Gene Ontology (GO), and Kyoto Encyclopedia of Genes and Genomes (KEGG). Then we used the STRING to establish protein-protein interactions and the Cytoscape tool to establish miRNA-mRNA regulatory networks. Results: We identified 19 significant DEMs and 140 and 33 significantly upregulated and downregulated DEGs, respectively. Functional enrichment analysis indicated that significant DEGs were associated with the antigen processing and presentation, and B-cell receptor signaling pathways in BPD. Key DEGs, such as CD19, CD79B, MS4A1, and FCGR2B were selected as hub genes in PPI networks. Conclusions: In this study, we screened out 19 DEMs that might play important roles in the regulatory networks of BPD. Higher expression of miRNAs such as miR-15b-5p, hsa-miR-32-5p, miR-3613-3p, and miR33a-5p and lower expression of miRNAs such as miR-3960, miR-425-5p, and miR-3202 might be correlated with the process of BPD.
\end{abstract}

\section{Introduction}

Nowadays, with the development of the department of neonatology and advancements in medical technology, the number of premature infants surviving the neonatal period has been increasing. However, this has been accompanied by an increasing number of cases of bronchopulmonary dysplasia (BPD) in preterm infants (Jobe, 2011), a chronic lung disease with significant morbidity and mortality mainly due to long time oxygen therapy during the late canalicular or saccular phases of lung development. Accordingly, this might cause long term consequences in the neonates. Researches have shown that infants with BPD are easier to get respiratory infections and are more susceptible to frequent hospitalizations compared with healthy preterm and term infants in the first $2 \mathrm{y}$ of their life (Hilgendorff and O'Reilly, 2015). The pathogenesis of BPD has not been well elucidated yet, so identifying the mechanism that leads to the occurrence and development of $\mathrm{BPD}$ is an important issue.

The diagnostic criteria of BPD changes over time. The National Institute of Child Health and Human Development (NICHD) guidelines in 2001 imposed that the diagnose of BPD requires accumulated oxygen inhalation for at least 28 days after birth. While new NICHD guidelines in 2018 imposed that the diagnose of BPD should according to the oxygen concentration for at least 3 consecutive days at 36 weeks post-menstrual 
age (PMA). There is no question that BPD is a complex disease that develops progressively, has multiple causes, has a spectrum of severity and the diagnosis is relatively nonspecific which can differ between regions.

In recent years, the genetic background has been involved in the pathogenesis of BPD (Wang et al., 2019). Nowadays, we can disclose the molecular mechanism of BPD using high-throughput sequencing and high-resolution microarray analysis. Microarray analysis is a high-throughput, high-efficiency, and highautomation method that has been widely used in scientific research to provide the expression level of messenger RNA (mRNA) and noncoding RNAs (ncRNAs) in samples (Hung and Weng, 2017; Bolón-Canedo et al., 2019). Noted, ncRNAs mainly includes microRNA (miRNA), circular RNA (circRNA), and long noncoding RNA (lncRNA). Accordingly, miRNAs is a group of highly stable single-stranded RNA molecules that have been reported to play important roles in post-transcriptional gene regulation (Yang et al., 2013). They have been shown to regulate the mRNA and protein expression in various physiological and pathological processes, such as cellular differentiation, proliferation, apoptosis, angiogenesis, and cancer development (Hatley et al., 2010).

Many studies have revealed the role of miRNA during the pathogenesis of BPD (Silva et al., 2015).These miRNAs have been shown to regulate their targeted downstream genes through changes (over- or under-) in their expression. Lal et al. found that under-expression of miR-876-3p was connected with the development of BPD (Lal et al., 2018), Whereas, Zhang et al. found that over-expression of miRNA-206 contributed to the development of BPD through the up-regulation of fibronectin 1 (Zhang et al., 2013). However, despite ongoing research in this field, the molecular mechanism of BPD remains poorly understood. As far as we know, there have been few studies using microarray datasets to obtain key genes and construct miRNAmRNA regulatory pathways in BPD. Our study aimed to identify the key genes and differentially expressed miRNA (DEMs) and their underlying regulatory mechanisms in BPD using bioinformatic methods.

\section{Materials and Methods}

\subsection{Microarray data}

GEO (http://www.ncbi.nlm.nih.gov/geo) is a public functional genomics data repository containing arrayand sequence-based data (Clough and Barrett, 2016). The GSE108754 gene expression profiles and the GSE108755 miRNA expression profiles were downloaded from GEO. A total of 20 premature infants with BPD and 20 non-BPD age-matched controls were enrolled from NICU in Shanghai Children Hospital from January 2015 to December 2016. BPD is diagnosed according to the National Institute of Child Health and Human Development (NICHD) guidelines in 2001. There was no significant difference in general clinical data between the two groups. Peripheral blood samples were collected. 5 infants with BPD and 6 infants without BPD were randomly selected for screening DEMs and DEGs, the others were used to verify. The array data were based on the GPL17107 Exiqon miRCURY LNA microRNA Array - hsa, mmu \& rno (Bethesda, MD, USA) and GPL13497 Agilent-026652 Whole Human Genome Microarray 4x44K v2 (Probe Name version) (Palo Alto, CA, USA). Moreover, we compared the results of GSE125873 and GSE32472 with our analysis.

\subsection{Identification of differentially expressed miRNAs and differentially expressed genes}

We compared samples from infants with BPD with those from normal preterm infants in GSE108755 and GSE108754 to identify DEMs and DEGs. The comparison was conducted using a Limma R package based on NetworkAnalyst 3.0 (https://www.networkanalyst.ca/NetworkAnalyst/home.xhtml) (Zhou et al., 2019). The "P value $<0.05$ " and "|log2 fold change (FC)| [?] 1" indexes were set as filtering criteria to screen for significant DEMs. False-positive results could be corrected using the adjusted $\mathrm{P}$ value from the BenjaminiHochberg method, and the "adjusted $\mathrm{P}$ value $<0.05$ " and "|log2 fold change (FC)|[?]1" indexes were used as primary filtering criteria to screen for DEGs.

\subsection{Identification of miRNA targets}

The MiRWalk 2.0 database (http://mirwalk.umm.uni-heidelberg.de/) integrates some miRNA databases and provides a huge amount of predicted and experimentally validated information about the binding 
targets of miRNAs (Sticht et al., 2018). We obtained the results of significant DEMs and their target mRNAs from the MiRwalk 2.0 retrieval system. Then, we used an online webtool, Venn diagrams (http://bioinformatics.psb.ugent.be/webtools/Venn/) to screen the genes from the intersection between the DEGs obtained from the GEO dataset and the targeted mRNAs predicted by MiRwalk 2.0. These intersected genes were selected as significant DEGs.

\subsection{Functional enrichment analysis}

Gene ontology (GO) is a tool used for gene annotation that functions through the utilization of a defined, structured, and controlled vocabulary, including 3 categories, namely biological processes (BP), cellular components (CC), and molecular functions (MF) (Ashburner et al., 2000). The Kyoto Encyclopedia of Genes and Genomes (KEGG) is a database used to assign sets of DEGs to specific pathways (Kanehisa and Goto, 2000). The Database for Annotation, Visualization, and Integrated Discovery (DAVID) (https://david.ncifcrf.gov/) is an online database that offers gene enrichment analysis and functional annotation clustering from various genomic resources (Huang et al., 2009). GO and KEGG pathway analysis on significant DEGs was conducted using the DAVID database. The species selected was Homo sapiens , and the "adjusted P value $<0.05$ " was considered to be of statistical significance.

\section{5 Analysis of protein-protein interaction (PPI) networks}

The Search Tool for the Retrieval of Interacting Genes/Proteins (STRING) (https://stringdb.org/cgi/input.pl) is an online database, which can predict interactions of proteins by neighborhood, gene fusion, co-occurrence, co-expression experiments, databases, and text-mining (Szklarczyk et al., 2015). Significant DEGs were mapped into PPIs and a combined score of $>0.4$ was set as a threshold value in this study. More specifically, nodes with higher degrees of interaction were considered as hub nodes.

\subsection{Analysis of miRNA-mRNA regulatory networks}

TargetScan (http://www.targetscan.org/) is a database that can be used to predict miRNA targets by matching conserved 8 mer and 7 mer sites with the seed region of an input miRNA (Lewis et al., 2005). MiRDB (http://www.mirdb.org/) is an online database that predicts miRNA target genes based on high throughput sequencing data (Wong and Wang, 2015). In addition, miRTarBase (http://mirtarbase.mbc.nctu.edu.tw/) is an experimentally-verified miRNA target gene database (Chou et al., 2016). Target genes that were verified by at least 1 of the 3 databases (TargetScan, miRDB, or miRTarBase) were selected as final sreened-out target genes for significant DEMs, and were used to build a miRNA-mRNA pairing file. Finally, the miRNAmRNA regulatory network was constructed using Cytoscape, an open source bioinformatics software program used to visualize molecular interaction networks (Shannon et al., 2003).

\section{Results}

\subsection{Identification of differentially expressed miRNAs / differentially expressed genes}

We compared the miRNA and mRNA expression profiles of the whole blood samples obtained from the 5 patients with BPD and 6 healthy controls. After statistical validation, the results of NetworkAnalyst analysis showed that there were 19 significant DEMs identified. Among them, 10 DEMs were upregulated, whereas 9 DEMs were downregulated (Table 1). In total, 207 DEGs were identified, including 168 upregulated and 39 downregulated (Appendix 1). The distribution of whole DEGs is shown in volcano plots (Fig. 1B), while the top 50 DEGs are shown in a heatmap (Fig. 1A).

\subsection{Identification of miRNA targets}

Using the MiRWalk 2.0 validated-target miRNA-gene retrieval system, we obtained a collection of candidate genes of the 19 significant DEMs. The intersection number between these candidate genes and the DEGs screened out by NetworkAnalyst was 173, with 140 genes being upregulated and 33 genes downregulated (Appendix 2). Therefore, the 140 upregulated and 33 downregulated genes were collected as candidates for the final profiles of significant DEGs, including ADM, WNT3, WNT16, ZNF532 and ZNF608. 


\subsection{GO and KEGG enrichment}

The GO was used to identify key biological functions corresponding to 1 of 3 term categories, namely, biological process, cellular component, and molecular function. Results showed that the most significant GO terms in each term category for 173 significant DEGs were "antigen processing and presentation of peptide or polysaccharide antigen via MHC class II", "MHC class II protein complex", and "MHC class II receptor activity" (Table 2, Fig. 2A). Furthermore, the identified significantly enriched KEGG pathways of significant DEGs were demonstrated to be about infection, inflammation, and immune response (Table 2, Fig. 2B). Among them, virus infection, antigen processing and presentation, and B cell receptor were identified as those that might play an important role in the pathogenesis of BPD.

Similarly, the results of GSE32472 were analyzed by Pietrzyk et al. suggested that the signaling pathway of the hematopoietic cell lineage, allograft rejection, asthma, intestinal immune network for IgA production, and cell adhesion molecules (CAMs) were also involved in BPD (Pietrzyk et al., 2013). Moreover, the hematopoietic cell lineage signaling pathway could be obtained in all 3 measurements in their study. Regarding the results of GSE125873 which were analyzed by Ryan et al., they also found the signaling pathway of CAMs, phagosome, systemic lupus erythematosus, and leishmaniasis. In addition, they also obtained the processes of "antigen processing and presentation of exogenous peptide antigen via MHC class II", "antigen processing and presentation of peptide or polysaccharide antigen via MHC class II" and "MHC class II receptor activity" in the terms of the GO-BP category (Ryan et al., 2019).

\subsection{PPI network and hub gene}

The resulting PPI networks were constructed by 173 significant DEGs (Fig.3), constituted by 166 nodes and 334 edges in total. In a PPI network, the genes which have more edges are known to play more important roles (like a seed). The "Degree" was used to count edge numbers of every gene in a PPI network. The top 10 genes, which were identified as hub genes, are shown in Table 3. Interestingly, all of 10 hub genes, such as CD19, CD79B, CD74 and CD72 were observed to be upregulated in the blood of infants with BPD compared with normal preterm infants. These genes were also shown to be related to the downstream signaling events of the B cell receptors and of the hematopoietic cell lineage.

\section{5 miRNA-mRNA regulatory network}

Target genes that were verified by at least 1 of the 3 databases (TargetScan, miRDB, or miRTarBase) were selected as final screened-out target genes for DEMs. As illustrated in Figure 4, 1 miRNA can target 1 or more mRNAs to regulate their functions. Moreover, 1 or more miRNAs might interact with the same mRNA. For example, miR-4286, miR-425-5p and miR-3490-5p were shown to interact with the AF4/FMR2 family member 3 (AFF3). Noted, AFF3 encodes a tissue-restricted nuclear transcriptional activator that is preferentially expressed and located in the nucleus of $\mathrm{B}$ cells and might play a role in lymphoid development and oncogenesis (Shi et al., 2018). Overall, there were 25 mRNA nodes discovered, including BCL11A, CDK14, MOB3B, and TCF4, which were noted to interplay with at least 2 miRNAs.

\section{Discussion}

Bronchopulmonary dysplasia is one of the most common complications arising in preterm infants, especially in those born underweight and those of small gestation weeks. It has been reported that up to 70 $\%$ of babies born before 26 wk of gestation will develop BPD (Durrmeyer et al., 2012). The progression of $\mathrm{BPD}$ is known to be driven by multiple mechanisms, with the participation of a few important protein and signaling pathways, such as thec vascular endothelial growth factor (VEGF), interleukin (IL), and phosphatidyl inositol-3-enzyme-serine/threonine kinase (PI3K-AKT) signaling pathway(Kalikkot Thekkeveedu et al., 2017). Therefore, it is important to clarify the pathophysiology of BPD and discover means of early diagnosis and treatment-related biomarkers. Bioinformatics analysis and efficient microarray might be conducive to our understanding of the molecular mechanisms of disease occurrence and development, thus helping the exploration of genetic alternations and identification of underlying diagnostic biomarkers.

In this study, we screened out 19 significant DEMs, of which 10 were shown to be upregulated, whereas 9 
downregulated. Rcesults of functional enrichment analysis indicated that these significant DEGs were associated with the virus infection, antigen processing and presentation, B-cell receptor, phagosome, hematopoietic cell lineage, and CAMs signaling pathways in BPD. Among these signaling pathways, the CAMs pathway was also obtained in the analysis of GSE32472(Pietrzyk et al., 2013) and GSE125873(Ryan et al., 2019). In these 2 series they both identified the signaling pathway of T-cell receptor which was not obtained in our study, maybe due to the use of different grouping methods and sampling time. Key DEGs, such as CD19, CD22, CD72, CD74, MS4A1, and FCGR2B were identified as hub genes in PPI networks. Moreover, through the construction of the PPI network, we could recognize key genes, with which miRNAs might interplay with. Despite filtering the genes with the potential targets of the 19 significant DEMs, we could still identify 140 upregulated and 33 downregulated genes. Hence, considering the total number of DEMs, the enormous and complex miRNA-mRNA regulatory network could be unimaginable. The hub genes of a network are known to always be important, resembling "seeds", that could combine different signal pathways.

Furthermore, some of these DEGs were validated and found to be correlated with BPD. One of the DEGs, called adrenomedullin (ADM), which was found to be downregulated in our study, was shown to be regulated by hsa-miR-423-5p, hsa-miR-3940-5p, hsa-miR-767-5p, and hsa-miR-4301. Moreover, ADM has been shown to have potent angiogenic, anti-inflammatory, and antioxidant properties. Zhang et al. reported that ADM deficiency in human pulmonary microvascular endothelial cells (HPMEC) resulted in significantly increased the generation of hyperoxia-induced reactive oxygen species and cytotoxicity compared with ADM sufficient HPMEC, finally causing BPD (Zhang et al., 2015); however this finding remains to be validated. Likewise, WNT 3/16 were demonstrated to be upregulated in our study through many miRNAs, such as the underexpressed hsa-miR-767-5p, hsa-miR-5681b, hsa-miR-423-5p, hsa-miR-3940-5p, and hsa-miR-3960.The WNT family have also been found to be associated with the development of BPD. Hyperoxia is known to increase the expression of WNT2b, WNT 5a, WNT 9a, and WNT 16, and decrease the expression of WNT 4, WNT 10a, and WNT 11 (Lingappan and Savani, 2020). The WNT family of proteins includes a large number of members that control a variety of developmental processes, including cell fate, proliferation, polarity, and migration (Ota et al., 2016). Li et al. found that patients with BPD were characterized by an increased activity of Wnt/ $\beta$-catenin (Li et al., 2015). Similar to that, we also found an increased expression of WNT 16 in BPD, but the mechanisms by which WNT3 might cause BPD remain to be explored. Among the identified DEGs, we also found the upregulated TLR10, which was shown to be regulated by miRNA, such as the underexpressed hsa-miR-767-5p, hsa-miR-5681b, hsa-miR-423-5p,hsa-miR-3940-5p, and overexpressed hsa-miR-33a-5p, and hsa-miR-337-5p, to be enriched in the immune response process term of the GO-BP category. Toll Like Receptors (TLRs) are known to play an important role in regulating inflammation, maintaining mucosal homeostasis and preventing bacterial invasion (Akira et al., 2006). Rising evidence has implied that the TLR signaling pathway is the pivotal component of the pulmonary homeostatic program that abrogates lung inflammation and injury(Sampath et al., 2012). Many studies were aimed at Toll-interleukin 1 receptor domain-containing adaptor protein (TIRAP). Researchers have also found that TLR5 and TLR4 were associated with the occurrence of BPD via the MyD88-dependent pathway(Malash et al., 2016; Yao et al., 2017), and TLR10 was reported to active the TRL4 signaling pathway. So, TLR10 might also be related to the occurrence of BPD; another finding that requires confirmation.

In this study, we screened out 19 DEMs, suggested to modulate the expression of DEGs and contribute in the regulation of many pathways. Besides, we also found that single miRNA could interplay with many mRNA species, as well as that a single mRNA could also interplay with many miRNA species. Although most of them have not been reported in the mechanisms so far studied in patients with BPD, we could still obtain some information from existing studies. One such case was the hsa-miR-15b-5p, one of the identified DEMs in our study. Zhang et al. found that miR-15b-5p was upregulated in BPD mice(Zhang et al., 2011). Fu et al. have also reported that it has a protective action against oxidative stress in HGstimulated podocytes (Fu et al., 2019), while Ezzie et al. found that it was increased in patients with chronic obstructive pulmonary disease (COPD) and could potentiate the progression of fibrosis in lung tissues (Ezzie et al., 2012).As such, overexpression of the hsa-miR-15b-5p in the BPD blood samples in our results, might indicate a similar underlying association. Another DEM, hsa-miR-301a-3p, which was overexpressed in our 
study, was demonstrated to modulate DEGs, such as TLR10, CD72, and BMP3. This effect has been previously observed in animal experiments. The study by Dong et al. showed the overexpression of miR301a in a murine model of hyperoxia-induced bronchopulmonary dysplasia(Dong et al., 2012). Therefore, hsa-miR-301a-3p might also play a role in the mechanism of BPD development in infants, which remains to be validated.

Although we investigated the miRNA-mRNA regulatory pathway in BPD using bioinformatics methods, our study had some limitations that should be clarified. First, the samples were limited and might have led to high false-positive rates and one-sided results. Therefore, it is required to improve the detection power by integrating more datasets in future studies. Second, the source of microarray data was only from blood samples. Body fluids that could be noninvasively obtained in the clinic, such as sputum and urine might also contain miRNAs. Third, to confirm the mechanisms of hub genes related to BPD, it will be helpful to add some in vitro or in vivo experiments to validate our results. Forth, due to the absence of clinical data, we were unable to assess the relationship between DEMs and the severity of BPD. More clinical and demographic characteristics of infants with BPD are required for further analysis. Finally, experimental evidence, obtained from wet research, such as western blot, real-time PCR and immunohistochemistry assays are required to better delineate the role of hub genes and the potential mechanisms of BPD.

In this study, we found multiple miRNA-mRNA regulatory pathways and potential biomarkers of BPD, in line with our current knowledge of the pathophysiology of this disease. We believe that this hypothesisgenerating study offers a new insight into the molecular mechanisms of BPD through the and identification of several latent biomarkers that could be used toward its diagnosis and treatment.

\section{Conclusions}

we identified 19 significant DEMs that might play key roles in the regulatory networks of BPD. The higher expression of miRNAs, such as miR-15b-5p, hsa-miR-32-5p, miR-3613-3p, and miR-33a-5p, and the lower expression of miRNAs, such as miR-3960, miR-425-5p, and miR-3202 might be related to the occurrence and development of BPD. However, we still need further experimental studies to test our results.

\section{Disclosure Statement}

No competing financial interests exist.

\section{Acknowledgements}

We gratefully acknowledge the teams of Cai et al., Ryan et al. and Pietrzyk et al. for sharing their data on GEO database.

\section{References}

Akira, S., Uematsu, S., and Takeuchi, O. (2006). Pathogen recognition and innate immunity. Cell 124(4), 783-801.

Ashburner, M., Ball, C.A., Blake, J.A., Botstein, D., Butler, H., Cherry, J.M., et al. (2000). Gene ontology: tool for the unification of biology. The Gene Ontology Consortium. Nature genetics25(1), 25-29.

Bolón-Canedo, V., Alonso-Betanzos, A., López-de-Ullibarri, I., and Cao, R. (2019). Challenges and Future Trends for Microarray Analysis. Methods in molecular biology (Clifton, N.J.) 1986,283-293. doi: 10.1007/9781-4939-9442-7_14.

Chou, C.-H., Chang, N.-W., Shrestha, S., Hsu, S.-D., Lin, Y.-L., Lee, W.-H., et al. (2016). miRTarBase 2016: updates to the experimentally validated miRNA-target interactions database. Nucleic acids research 44(D1), D239-D247. doi: 10.1093/nar/gkv1258.

Clough, E., and Barrett, T. (2016). The Gene Expression Omnibus Database. Methods in molecular biology (Clifton, N.J.) 1418. doi: 10.1007/978-1-4939-3578-9_5. 
Dong, J., Carey, W.A., Abel, S., Collura, C., Jiang, G., Tomaszek, S., et al. (2012). MicroRNA-mRNA interactions in a murine model of hyperoxia-induced bronchopulmonary dysplasia. BMC Genomics13, 204. doi: 10.1186/1471-2164-13-204.

Durrmeyer, X., Kayem, G., Sinico, M., Dassieu, G., Danan, C., and Decobert, F. (2012). Perinatal risk factors for bronchopulmonary dysplasia in extremely low gestational age infants: a pregnancy disorderbased approach. The Journal of pediatrics 160(4). doi: 10.1016/j.jpeds.2011.09.025.

Ezzie, M.E., Crawford, M., Cho, J.-H., Orellana, R., Zhang, S., Gelinas, R., et al. (2012). Gene expression networks in COPD: microRNA and mRNA regulation. Thorax 67(2), 122-131. doi: 10.1136/thoraxjnl-2011200089 .

Fu, Y., Wang, C., Zhang, D., Chu, X., Zhang, Y., and Li, J. (2019). miR-15b-5p ameliorated high glucoseinduced podocyte injury through repressing apoptosis, oxidative stress, and inflammatory responses by targeting Sema3A. Journal of cellular physiology234(11), 20869-20878. doi: 10.1002/jcp.28691.

Hatley, M.E., Patrick, D.M., Garcia, M.R., Richardson, J.A., Bassel-Duby, R., van Rooij, E., et al. (2010). Modulation of K-Ras-dependent lung tumorigenesis by MicroRNA-21. Cancer cell18(3), 282-293. doi: 10.1016/j.ccr.2010.08.013.

Hilgendorff, A., and O'Reilly, M.A. (2015). Bronchopulmonary dysplasia early changes leading to long-term consequences. Frontiers in medicine 2, 2. doi: 10.3389/fmed.2015.00002.

Huang, D.W., Sherman, B.T., and Lempicki, R.A. (2009). Systematic and integrative analysis of large gene lists using DAVID bioinformatics resources. Nature protocols 4(1), 44-57. doi: 10.1038/nprot.2008.211.

Hung, J.-H., and Weng, Z. (2017). Analysis of Microarray and RNA-seq Expression Profiling Data. Cold Spring Harbor protocols 2017(3). doi: 10.1101/pdb.top093104.

Jobe, A.H. (2011). The new bronchopulmonary dysplasia. Current opinion in pediatrics 23(2), 167-172. doi: 10.1097/MOP.0b013e3283423e6b.

Kalikkot Thekkeveedu, R., Guaman, M.C., and Shivanna, B. (2017). Bronchopulmonary dysplasia: A review of pathogenesis and pathophysiology. Respiratory medicine 132, 170-177. doi: 10.1016/j.rmed.2017.10.014.

Kanehisa, M., and Goto, S. (2000). KEGG: kyoto encyclopedia of genes and genomes. Nucleic acids research 28(1), 27-30.

Lal, C.V., Olave, N., Travers, C., Rezonzew, G., Dolma, K., Simpson, A., et al. (2018). Exosomal microRNA predicts and protects against severe bronchopulmonary dysplasia in extremely premature infants. JCI insight 3(5). doi: 10.1172/jci.insight.93994.

Lewis, B.P., Burge, C.B., and Bartel, D.P. (2005). Conserved seed pairing, often flanked by adenosines, indicates that thousands of human genes are microRNA targets. Cell 120(1), 15-20.

Li, J., Yu, K.-H., Oehlert, J., Jeliffe-Pawlowski, L.L., Gould, J.B., Stevenson, D.K., et al. (2015). Exome Sequencing of Neonatal Blood Spots and the Identification of Genes Implicated in Bronchopulmonary Dysplasia. American journal of respiratory and critical care medicine 192(5), 589-596. doi: 10.1164/rccm.2015010168OC.

Lingappan, K., and Savani, R.C. (2020). The Wnt Signaling Pathway and the Development of Bronchopulmonary Dysplasia. American journal of respiratory and critical care medicine . doi: 10.1164/rccm.2020020277ED.

Malash, A.H., Ali, A.A., Samy, R.M., and Shamma, R.A. (2016). Association of TLR polymorphisms with bronchopulmonary dysplasia. Gene 592(1), 23-28. doi: 10.1016/j.gene.2016.07.049.

Ota, C., Baarsma, H.A., Wagner, D.E., Hilgendorff, A., and Königshoff, M. (2016). Linking bronchopulmonary dysplasia to adult chronic lung diseases: role of WNT signaling. Molecular and cellular pediatrics 3(1), 
34.

Pietrzyk, J.J., Kwinta, P., Wollen, E.J., Bik-Multanowski, M., Madetko-Talowska, A., Günther, C.-C., et al. (2013). Gene expression profiling in preterm infants: new aspects of bronchopulmonary dysplasia development. PloS one 8(10), e78585. doi: 10.1371/journal.pone.0078585.

Ryan, F.J., Drew, D.P., Douglas, C., Leong, L.E.X., Moldovan, M., Lynn, M., et al. (2019). Changes in the Composition of the Gut Microbiota and the Blood Transcriptome in Preterm Infants at Less than 29 Weeks Gestation Diagnosed with Bronchopulmonary Dysplasia. mSystems4(5). doi: 10.1128/mSystems.00484-19.

Sampath, V., Garland, J.S., Le, M., Patel, A.L., Konduri, G.G., Cohen, J.D., et al. (2012). A TLR5 (g.1174C $>\mathrm{T}$ ) variant that encodes a stop codon (R392X) is associated with bronchopulmonary dysplasia. Pediatric pulmonology 47(5), 460-468. doi: 10.1002/ppul.21568.

Shannon, P., Markiel, A., Ozier, O., Baliga, N.S., Wang, J.T., Ramage, D., et al. (2003). Cytoscape: a software environment for integrated models of biomolecular interaction networks. Genome research13(11), 2498-2504.

Shi, Y., Zhao, Y., Zhang, Y., AiErken, N., Shao, N., Ye, R., et al. (2018). AFF3 upregulation mediates tamoxifen resistance in breast cancers. Journal of experimental \& clinical cancer research : CR37(1), 254. doi: 10.1186/s13046-018-0928-7.

Silva, D.M.G., Nardiello, C., Pozarska, A., and Morty, R.E. (2015). Recent advances in the mechanisms of lung alveolarization and the pathogenesis of bronchopulmonary dysplasia. American journal of physiology. Lung cellular and molecular physiology 309(11),L1239-L1272. doi: 10.1152/ajplung.00268.2015.

Sticht, C., De La Torre, C., Parveen, A., and Gretz, N. (2018). miRWalk: An online resource for prediction of microRNA binding sites. PloS one 13(10), e0206239. doi: 10.1371/journal.pone.0206239.

Szklarczyk, D., Franceschini, A., Wyder, S., Forslund, K., Heller, D., Huerta-Cepas, J., et al. (2015). STRING v10: protein-protein interaction networks, integrated over the tree of life. Nucleic acids research 43(Database issue), D447-D452. doi: 10.1093/nar/gku1003.

Wang, J., Yin, J., Wang, X., Liu, H., Hu, Y., Yan, X., et al. (2019). Changing expression profiles of mRNA, lncRNA, circRNA, and miRNA in lung tissue reveal the pathophysiological of bronchopulmonary dysplasia (BPD) in mouse model. J Cell Biochem 120(6), 9369-9380. doi: 10.1002/jcb.28212.

Wong, N., and Wang, X. (2015). miRDB: an online resource for microRNA target prediction and functional annotations. Nucleic acids research 43(Database issue), D146-D152. doi: 10.1093/nar/gku1104.

Yang, Y., Qiu, J., Kan, Q., Zhou, X.G., and Zhou, X.Y. (2013). MicroRNA expression profiling studies on bronchopulmonary dysplasia: a systematic review and meta-analysis. Genetics and molecular research : GMR12(4), 5195-5206. doi: 10.4238/2013.October.30.4.

Yao, L., Shi, Y., Zhao, X., Hou, A., Xing, Y., Fu, J., et al. (2017). Vitamin D attenuates hyperoxia-induced lung injury through downregulation of Toll-like receptor 4. International journal of molecular medicine 39(6), 1403-1408. doi: 10.3892/ijmm.2017.2961.

Zhang, S., Patel, A., Moorthy, B., and Shivanna, B. (2015). Adrenomedullin deficiency potentiates hyperoxic injury in fetal human pulmonary microvascular endothelial cells. Biochemical and biophysical research communications 464(4), 1048-1053. doi: 10.1016/j.bbrc.2015.07.067.

Zhang, X., Peng, W., Zhang, S., Wang, C., He, X., Zhang, Z., et al. (2011). MicroRNA expression profile in hyperoxia-exposed newborn mice during the development of bronchopulmonary dysplasia. Respiratory care 56(7), 1009-1015. doi: 10.4187/respcare.01032.

Zhang, X., Xu, J., Wang, J., Gortner, L., Zhang, S., Wei, X., et al. (2013). Reduction of microRNA-206 contributes to the development of bronchopulmonary dysplasia through up-regulation of fibronectin 1.PloS one 8(9), e74750. doi: 10.1371/journal.pone.0074750. 
Zhou, G., Soufan, O., Ewald, J., Hancock, R.E.W., Basu, N., and Xia, J. (2019). NetworkAnalyst 3.0: a visual analytics platform for comprehensive gene expression profiling and meta-analysis. Nucleic acids research 47(W1), W234-W241. doi: 10.1093/nar/gkz240.

Table 1. Significant DEMs in infants with BPD compared with normal preterm infants.

\begin{tabular}{llll}
\hline miRNA ID & Expression in BPD & $\log F C$ & P.Value \\
\hline hsa-miR-15b-5p & Up regulation & 12.911 & 0.003439 \\
hsa-miR-32-5p & Up regulation & 4.9954 & 0.003765 \\
hsa-miR-3613-3p & Up regulation & 1.1189 & 0.010372 \\
hsa-miR-33a-5p & Up regulation & 2.0471 & 0.012098 \\
hsa-miR-4764-3p & Up regulation & 1.1531 & 0.015397 \\
hsa-miR-301a-3p & Up regulation & 1.4162 & 0.020749 \\
hsa-miR-30c-5p & Up regulation & 3.52 & 0.021143 \\
hsa-miR-3924 & Up regulation & 1.1278 & 0.039454 \\
hsa-miR-337-5p & Up regulation & 1.3447 & 0.048196 \\
hsa-miR-30b-5p & Up regulation & 3.4964 & 0.049496 \\
hsa-miR-3960 & Down regulation & -1.1572 & 0.001596 \\
hsa-miR-425-5p & Down regulation & -1.6406 & 0.005113 \\
hsa-miR-3202 & Down regulation & -1.6424 & 0.005203 \\
hsa-miR-5681b & Down regulation & -2.4807 & 0.01101 \\
hsa-miR-4286 & Down regulation & -2.0298 & 0.014811 \\
hsa-miR-767-5p & Down regulation & -3.267 & 0.027295 \\
hsa-miR-3940-5p & Down regulation & -2.7115 & 0.028662 \\
hsa-miR-4301 & Down regulation & -6.5082 & 0.02986 \\
hsa-miR-423-5p & Down regulation & -1.6656 & 0.043209 \\
\hline
\end{tabular}

Table 2. GO and KEGG pathway analysis

\begin{tabular}{|c|c|c|}
\hline Category & Term & Term \\
\hline $\mathrm{BP}$ & $\mathrm{BP}$ & GO:0002504 antigen processing and presentation of peptide or polysaccharide antigen via MHC class \\
\hline $\mathrm{BP}$ & $\mathrm{BP}$ & GO:0006955 immune response \\
\hline $\mathrm{BP}$ & $\mathrm{BP}$ & GO:0019886 antigen processing and presentation of exogenous peptide antigen via MHC class II \\
\hline $\mathrm{BP}$ & $\mathrm{BP}$ & GO:0019882 $\sim$ antigen processing and presentation \\
\hline $\mathrm{BP}$ & $\mathrm{BP}$ & GO:0031295 $\mathrm{T}$ cell costimulation \\
\hline $\mathrm{BP}$ & $\mathrm{BP}$ & GO:0060333 interferon-gamma-mediated signaling pathway \\
\hline $\mathrm{CC}$ & $\mathrm{CC}$ & GO:0042613 MHC class II protein complex \\
\hline $\mathrm{CC}$ & $\mathrm{CC}$ & GO:0071556 integral component of lumenal side of endoplasmic reticulum membrane \\
\hline $\mathrm{CC}$ & $\mathrm{CC}$ & GO:0030666〜endocytic vesicle membrane \\
\hline $\mathrm{CC}$ & $\mathrm{CC}$ & GO:0012507 ER to Golgi transport vesicle membrane \\
\hline $\mathrm{CC}$ & $\mathrm{CC}$ & GO:0030658 transport vesicle membrane \\
\hline $\mathrm{CC}$ & $\mathrm{CC}$ & GO:0030669^ clathrin-coated endocytic vesicle membrane \\
\hline $\mathrm{CC}$ & $\mathrm{CC}$ & GO:0032588 trans-Golgi network membrane \\
\hline $\mathrm{CC}$ & $\mathrm{CC}$ & GO:0005886 plasma membrane \\
\hline $\mathrm{CC}$ & $\mathrm{CC}$ & GO:0005765 lysosomal membrane \\
\hline $\mathrm{CC}$ & $\mathrm{CC}$ & GO:0010008 endosome membrane \\
\hline $\mathrm{CC}$ & $\mathrm{CC}$ & GO:0005887 integral component of plasma membrane \\
\hline MF & MF & GO:0032395 MHC class II receptor activity \\
\hline MF & MF & GO:0023026 MHC class II protein complex binding \\
\hline MF & MF & GO:0042605 peptide antigen binding \\
\hline
\end{tabular}




\begin{tabular}{lll}
\hline Category & Term & Term \\
\hline KEGG & KEGG & hsa05166: HTLV-I infection \\
KEGG & KEGG & hsa05150: Staphylococcus aureus infection \\
KEGG & KEGG & hsa04672: Intestinal immune network for IgA production \\
KEGG & KEGG & hsa05310: Asthma \\
KEGG & KEGG & hsa05332: Graft-versus-host disease \\
KEGG & KEGG & hsa05330: Allograft rejection \\
KEGG & KEGG & hsa04940: Type I diabetes mellitus \\
KEGG & KEGG & hsa05321: Inflammatory bowel disease (IBD) \\
KEGG & KEGG & hsa05140: Leishmaniasis \\
KEGG & KEGG & hsa05320: Autoimmune thyroid disease \\
KEGG & KEGG & hsa04612: Antigen processing and presentation \\
KEGG & KEGG & hsa05416: Viral myocarditis \\
KEGG & KEGG & hsa04514: Cell adhesion molecules (CAMs) \\
KEGG & KEGG & hsa05323: Rheumatoid arthritis \\
KEGG & KEGG & hsa04145: Phagosome \\
KEGG & KEGG & hsa05145: Toxoplasmosis \\
KEGG & KEGG & hsa04662: B cell receptor signaling pathway \\
KEGG & KEGG & hsa05322: Systemic lupus erythematosus \\
KEGG & KEGG & hsa05152: Tuberculosis \\
KEGG & KEGG & hsa05168: Herpes simplex infection \\
KEGG & KEGG & hsa05169: Epstein-Barr virus infection \\
KEGG & KEGG & hsa05164: Influenza A \\
KEGG & KEGG & hsa04640: Hematopoietic cell lineage \\
\hline
\end{tabular}

Table 3. Top 10 hub genes in the PPI network

\begin{tabular}{llll}
\hline Gene Symbol & Full gene name & Expression in BPD & Degree \\
\hline CD19 & CD19 molecule & up regulation & 29 \\
CD79B & CD79b molecule & up regulation & 27 \\
MS4A1 & membrane spanning 4-domains A1 & up regulation & 21 \\
FCGR2B & Fc fragment of IgG receptor IIb & up regulation & 19 \\
CD22 & CD22 molecule & up regulation & 18 \\
CD74 & CD74 molecule & up regulation & 18 \\
BLK & BLK proto-oncogene, Src family tyrosine kinase & up regulation & 17 \\
CXCR5 & C-X-C motif chemokine receptor 5 & up regulation & 16 \\
IGLL5 & immunoglobulin lambda like polypeptide 5 & up regulation & 16 \\
CD72 & CD72 molecule & up regulation & 15 \\
\hline
\end{tabular}


A
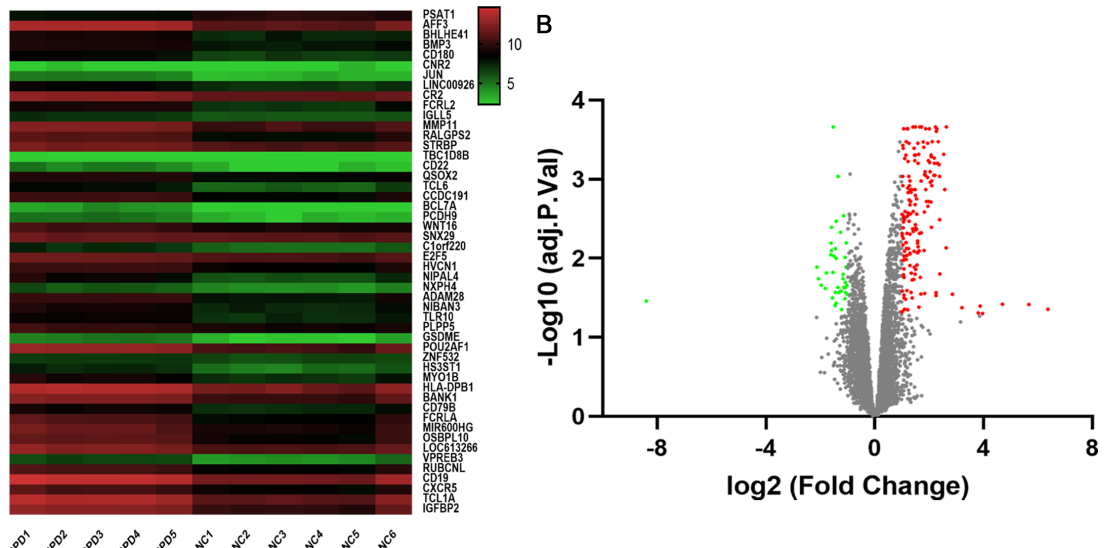

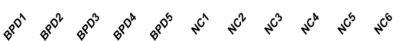

A

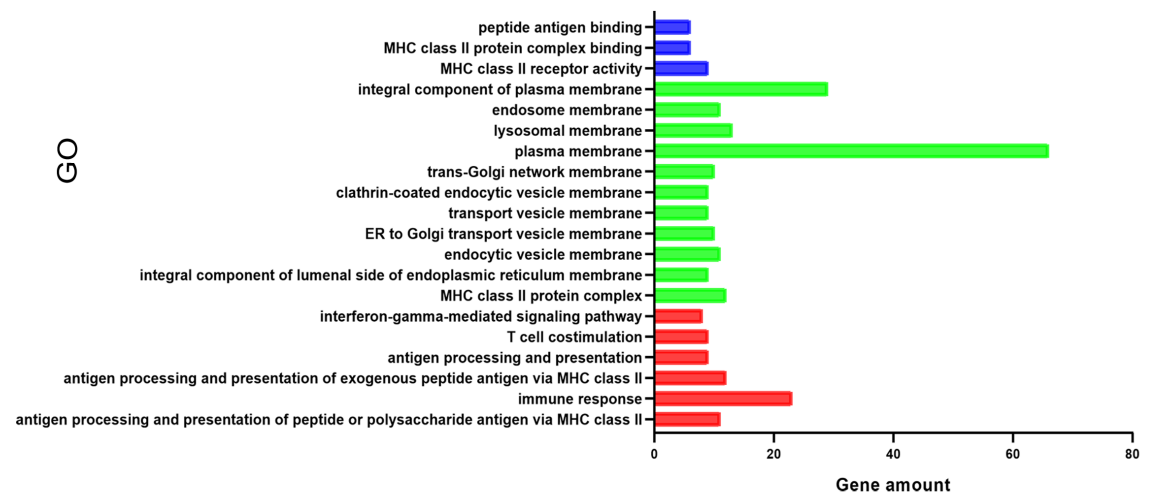

B

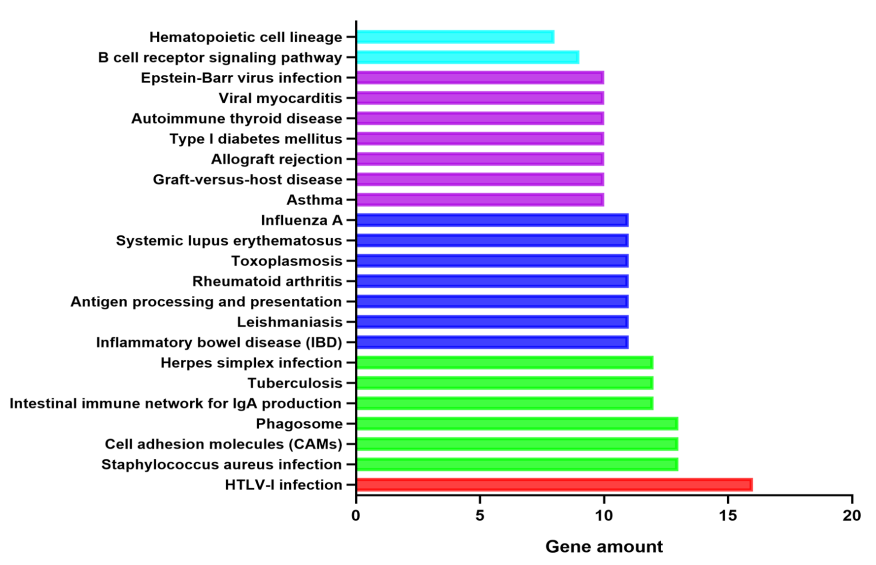



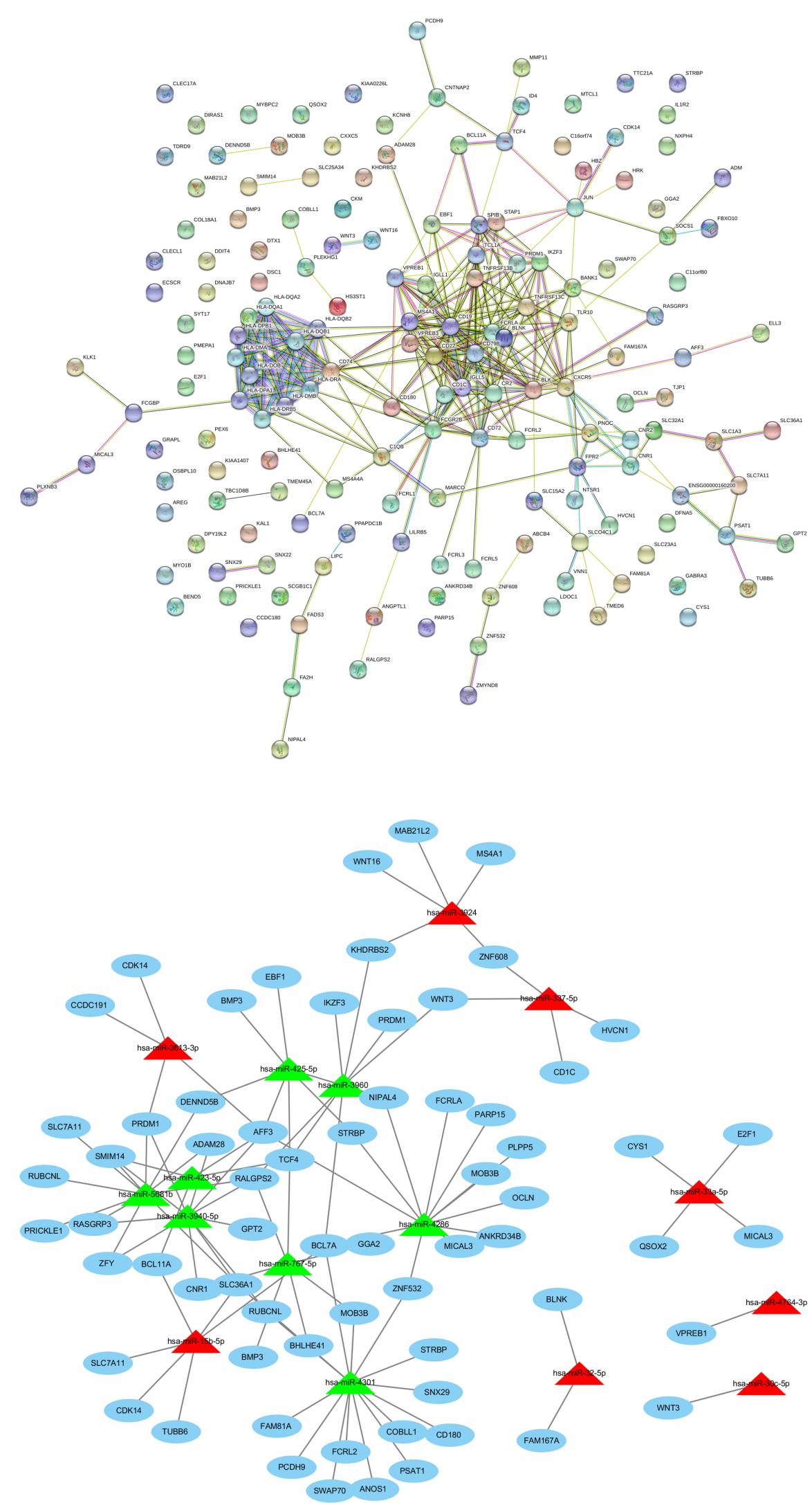

12 